\title{
Sight versus sound
}

A new study of spatial localization of audiovisual stimuli has shown that the 'ventriloquist effect' is a function of nearoptimal integration of visual and auditory inputs.

Ventriloquism is a phenomenon whereby we inaccurately perceive a voice as emanating from a spatially displaced source; for example, from the lips of a ventriloquist's dummy rather than from those of the ventriloquist. The effect was originally thought to be the product of voice projection techniques perfected by the performer, but recent hypotheses have suggested that it results from the domination of hearing by vision.

Alais and Burr tested this assertion by having observers localize visual and audio stimuli — brief 'blobs' of light or 'clicks' of sound - in space. Unimodal thresholds were established by presenting these stimuli separately and asking observers to indicate which of the two stimuli appeared more to the left. Subsequently, blobs and clicks were presented simultaneously in one of two modes. In 'conflict' mode, blobs and clicks were spatially displaced from each other; in 'non-conflict' mode, the stimuli were equally displaced to the left or right of centre.

The ability of subjects to localize the stimuli depended on the size and clarity of the blobs. When visual localization was good, vision 'captured' sound, as in the classic ventriloquist effect. However, the reverse was true when visual stimuli were blurred and therefore poorly localized. In this case, the subjects perceived the blob as closer to the correct location of the click, rather than vice versa. In all six subjects, bimodal localization was more precise than either form of unimodal localization. Based on these data, the authors propose a model in which visual and auditory inputs are optimally combined to minimize variance and improve spatial localization.

Suzanne Farley

\section{Q) References and links} ORIGINAL RESEARCH PAPER Alais, D. \& Burr, D. The ventriloquist effect results from near-optimal bimodal integration. Curr. Biol. 14, 257-262 (2004) FURTHER READING Ernst, M. O. \& Banks, M. S. Humans integrate visual and haptic information in a statistically optimal fashion. Nature 415, 429-433 (2002)

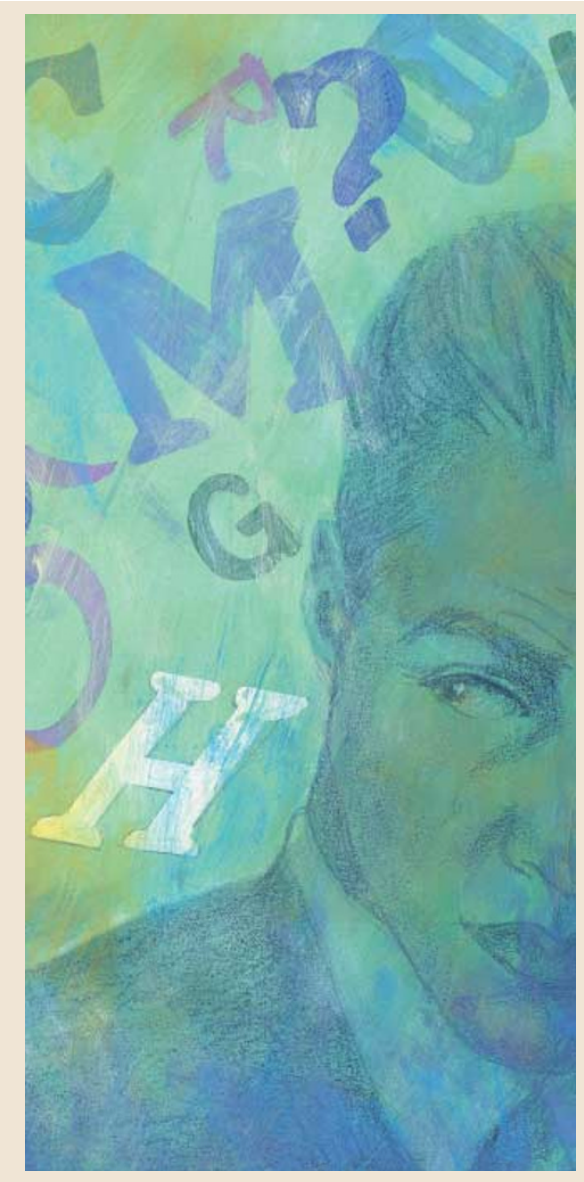

\section{A convergence point in MS pathology}

The enzyme cytosolic phospholipase $\mathrm{A}_{2}$ ( CPLA $_{2}$ ) may have a key role in the pathogenesis of multiple sclerosis (MS), according to a new study published in Neuron. The findings, made by Kalyvas and David in an animal model of the condition, could lead to better treatments for MS patients.

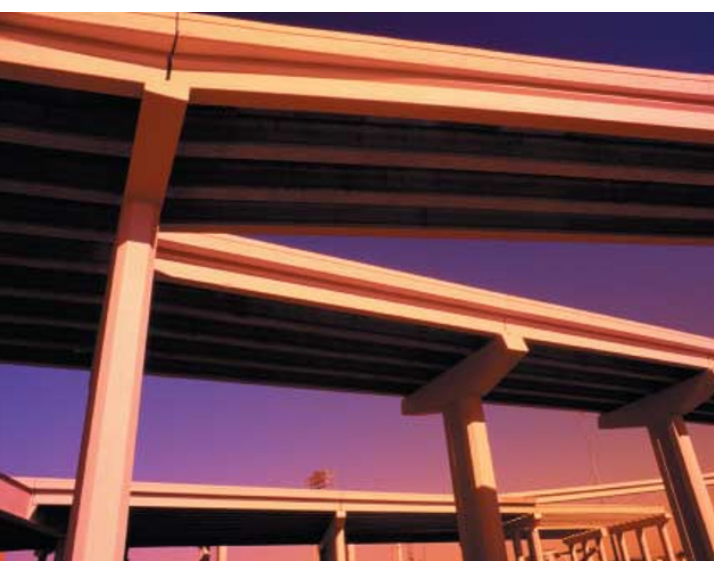

MS is an inflammatory, demyelinating disease of the central nervous system (CNS). It is thought to involve an autoimmune response in which myelin-reactive cells enter the CNS and initiate the disease. Although the aetiology and pathogenesis of the disorder are still not fully understood, it seems that various causative factors trigger a common mechanism, resulting in the classical pathology of MS - immune cell infiltration into the CNS, a complex inflammatory cascade, and demyelination and axonal damage.

Kalyvas and David looked for a convergence point in the induction of these stereotypical pathologies. They focused on $\mathrm{CPLA}_{2}$ for two main reasons: first, because many pro-inflammatory chemokines and cytokines produced in the early stages of MS can induce PLA ; and second, because metabolic products of PLA $_{2}$, including arachidonic acid (AA) and lysophosphatidylcholine (LPC), can mediate inflammation and demyelination.
The researchers examined mice with experimental autoimmune encephalomyelitis (EAE), an inflammatory, demyelinating condition induced by immunizing animals against myelin antigens. They found that $\mathrm{CPLA}_{2}$ was expressed at high levels in endothelial cells and immune cells at EAE lesions. Blocking the enzyme with an AA analogue led to a dramatic reduction in the onset and progression of EAE. The authors showed that $\mathrm{CPLA}_{2}$ was present at much reduced levels in mice treated with this inhibitor. What's more, they found that inhibiting $\mathrm{CPLA}_{2}$ in mice with EAE led to a decrease in the expression of many mediators of inflammation, including chemokines and cytokines that are known to induce the expression of CPLA $_{2}$ or to be induced by LPC.

These results suggest that CPLA ${ }_{2}$ could serve as the common link by which various factors trigger the inflammatory and axonal pathologies characteristic of EAE and MS. Studies of potent and specific inhibitors of $\mathrm{CPLA}_{2}$ as potential treatments for MS are now warranted.

Rebecca Craven, Senior Subeditor, Nature

\section{(4) References and links}

ORIGINAL RESEARCH PAPER Kalyvas, A. \& David, S.

Cytosolic phospholipase $A_{2}$ plays a key role in the

pathogenesis of multiple sclerosis-like disease. Neuron 41, 323-335 (2004) 